\title{
Blood Flow Restriction Alters Motor Unit Behavior During Resistance Exercise
}

\author{
Authors \\ Pedro Fatela1, 2 , Goncalo V. Mendonca ${ }^{3,2}$, António Prieto Veloso ${ }^{3,4}$, Janne Avela ${ }^{5}$, Pedro Mil-Homens ${ }^{3,2}$
}

\section{Affiliations}

1 Sport Sciences, Universidade Europeia, Lisboa, Portugal

2 Neuromuscular Research Lab, Faculdade de Motricidade Humana, Universidade de Lisboa, Estrada da Costa, 1499-002 Cruz Quebrada Dafundo, Portugal.

3 CIPER, Faculdade de Motricidade Humana, Universidade de Lisboa, Estrada da Costa, 1499-002, Cruz Quebrada Dafundo, Portugal.

4 Biomechanics Laboratory, Faculdade de Motricidade Humana, Universidade de Lisboa, Estrada da Costa, 1499-002 Cruz Quebrada Dafundo, Portugal.

5 Department of Biology of Physical Activity, University of Jyväskylä, Jyväskylä, Finland

\section{Key words}

KAATSU, muscle strength, intramuscular coordination, resistance exercise

accepted 25.03.2019

\section{Bibliography}

DOI https://doi.org/10.1055/a-0888-8816

Published online: 10.7.2019

Int J Sports Med 2019; 40: 555-562

(c) Georg Thieme Verlag KG Stuttgart - New York

ISSN 0172-4622

\section{Correspondence}

Prof. Goncalo V Mendonca, MS

Faculdade de Motricidade Humana,

Universidade de Lisboa,

Laboratory of Neuromuscular Function,

1495-688 Lisboa

Portugal

Tel.: + 351/933/206 691, Fax: + 351/214/149 236

gvmendonca@gmail.com

\section{ABSTRACT}

We aimed to determine whether blood flow restriction (BFR) alters the characteristics of individual motor units during lowintensity (LI) exercise. Eight men $(26.0 \pm 3.8 \mathrm{yrs})$ performed 5 sets of 15 knee extensions at $20 \%$ of one-repetition maximum (with and without BFR). Maximal isometric voluntary contractions (MVC) were performed before and after exercise to quantify force decrement. Submaximal isometric voluntary contractions were additionally performed for $18 \mathrm{~s}$, matching trapezoidal target-force trajectories at $40 \%$ pre-MVC. EMG activity was recorded from the vastus lateralis muscle. Then, signals were decomposed to extract motor unit recruitment threshold, firing rates and action potential amplitudes (MUAP). Force decrement was only seen after LI BFR exercise $(-20.5 \%$; $p<0.05)$. LI BFR exercise also induced greater decrements in the linear slope coefficient of the regression lines between motor unit recruitment threshold and firing rate (BFR: $-165.1 \pm 120.4$ vs. non-BFR: $-44.4 \pm 33.1 \%, p<0.05)$. Finally, there was a notable shift towards higher values of firing rate and MUAP amplitude post-LI BFR exercise. Taken together, our data indicate that LI BFR exercise increases the activity of motor units with higher MUAP amplitude. They also indicate that motor units with similar MUAP amplitudes become activated at higher firing rates post-LI BFR exercise.

\section{ABBREVIATIONS}

LI low-intensity exercise

LI BFR low-intensity exercise with blood flow restriction

\section{Introduction}

Past research indicates that blood flow restriction (BFR) enhances muscle activation during low-intensity (LI) exercise [1-7]. In fact, there is partial evidence that the acute neuromuscular response to LI BFR exercise is similar to that seen during non-BFR high-intensity resistance exercise [5-7]. Based on this, it has been suggested that both these methods of resistance training are equally effec- 
tive for enhancing the recruitment of high threshold motor units (MU) $[5,6,8]$. However, this hypothesis has not yet been empirically tested. It is merely supported by experimental studies in which the levels of muscular activation and neuromuscular fatigue were inferred from global parameters of surface electromyographic (EMG) signal amplitude [6, 8].

Automatic decomposition of surface EMG signals into their constituent motor unit action potential (MUAP) trains has recently been introduced $[9,10]$. The possibility of exploring the behavior of MUs non-invasively, by means of surface EMG decomposition, may allow further insight into the impact of LI BFR exercise on neuromuscular function. Previous studies have shown that, during fatiguing submaximal contractions, there is a decrease in the recruitment threshold of all MUs $[11,12]$. In parallel, as muscle fatigue becomes more severe, additional MUs are gradually recruited and the firing rate of the already active MUs also increases [11-16]. Theoretically, due to the nature of its metabolic demands, LI BFR exercise might also trigger such a pattern of MU behavior. This would serve as a compensatory strategy to counteract the progressive decline in force-generating capacity with muscle fatigue. Thus, we hypothesized that LI BFR exercise would induce higher firing rates in the low threshold MUs. Additionally, we hypothesized that high threshold MU (with lower firing rates) would be recruited more effectively following BFR vs. non-BFR exercise.

\section{Materials and Methods}

\section{Participants}

Eight healthy young men (age: $26.0 \pm 3.8 \mathrm{yrs}$; height: $173.6 \pm 9.1 \mathrm{~cm}$; body mass: $72.1 \pm 8.6 \mathrm{~kg}$ ), with minor experience in exercise training (not participating in any form of resistance or endurance training for the last 6 months), were recruited to participate in this study. The participants were informed of the experimental risks and were asked to sign an informed consent document before being enrolled in the first testing session. They were also instructed to maintain the same level of physical activity throughout the course of the study. Participants were all non-smokers and free from any known cardiovascular or metabolic diseases, as assessed by medical history. All participants were asked to avoid the intake of caffeine, alcohol and performing any form of exercise for at least $48 \mathrm{~h}$ before testing. Additionally, all participants were tested at approximately the same time of day $( \pm 2 \mathrm{~h})$. This study was approved by the Faculty Ethics Committee and complied with the principles set forth in the ethical standards of the journal [17].

\section{Study design}

After being familiarized with all testing procedures, participants were tested on two different conditions (in a randomized fashion) over a period of 1 week. Familiarization consisted of two sessions that served the purpose of ensuring adaptation to all testing procedures (including metronomic pacing and LI BFR exercise) and performing pre-exercise baseline measurements: (1) assessment of arterial occlusion pressure (AOP) for the right lower limb, and (2) determination of one-repetition maximum (1RM) for right knee extension. Each participant was tested on two different conditions of unilateral knee extension (LI exercise at 20\% 1RM vs. LI BFR ex- ercise at $20 \% 1 \mathrm{RM}$ ) using a modified isotonic leg extension machine (Technogym ${ }^{\circledR}$, Executive Line, Gambetolla, Italy), adapting a blocking system that allows isometric contractions in the proposed knee angles. All measurements were taken in the seated position, with the participants' arms in full extension and their hands holding the chair supports. A general warm-up was performed before each testing session: (1) 6 min of unloaded cycle ergometry (60-70 rpm), and (2) $2 \times 15$ isotonic knee extensions at $20 \% 1 \mathrm{RM}$. Then, the participants completed three different pre-evaluation time points before exercise ( $>$ Fig. 1): (1) maximal voluntary isometric contraction (MVC), (2) pre 1 - target-force trajectory at $40 \%$ MVC, and (3) pre 2 - target-force trajectory at $40 \%$ MVC. The same three evaluations were repeated in an inverse order after acute LI exercise with and without BFR. Measurements at pre 1 and post 2 time points were performed without BFR in both conditions. In the LI BFR condition, measurement of pre 2 and post 1 were made with BFR.

\section{One-repetition maximum}

1 RM was determined for right knee extension. Participants were asked to complete 1 repetition throughout a full range of motion $\left(90^{\circ}\right)$, with $1 \mathrm{RM}$ being defined as the maximal load that each participant mobilized in a single maximum dynamic knee extension. All participants received strong verbal encouragement in each attempt and 1 RM was always determined within 5 attempts (mean value: $78.9 \pm 19.9 \mathrm{~kg})$.

\section{Maximal voluntary contraction}

MVC was determined for right knee extension (before and after acute LI exercise with as well as without BFR). Knee joint angle was set at $\sim 70^{\circ}$ for all participants. MVC was measured in response to 3 isometric contractions with 3 s of duration, each separated by 1 min of recovery. Participants were instructed to exert their maximum force as hard as possible and MVC was defined as the maximum force obtained in 3 pre- and post-exercise attempts.

\section{Resistance exercise protocols}

Acute exercise (LI and LI BFR) included 5 sets of 15 repetitions for unilateral knee extensions performed at $20 \% 1$ RM, with 30 s of passive rest between sets ( $\triangleright$ Fig. 1). This training volume (i. e., 75 reps) has been used consistently in most studies examining the physiological responses to LI BFR resistance exercise [3, 18-22]. A metronome was used to control the cadence, with $1: 1 \mathrm{~s}$ for concentric and eccentric muscle contractions, respectively.

\section{Blood flow restriction}

BFR was set at $60 \%$ of AOP taken at resting conditions. AOP was determined during the familiarization session, over the right tibial artery, using a vascular Doppler probe (PD1 + Combi, Ultrasound Technologies Ltd, Caldicot, UK). BFR was elicited using a $13 \times 124 \mathrm{~cm}$ pneumatic cuff(SC12L Tourniquet Cuffs, D. E. Hokanson, Inc. Bellevue, WA) applied to the most proximal portion of the right thigh. To guarantee similar cuff placement between the familiarization and testing sessions, a photographic record was made for each participant. Each testing session was preceded by a structured protocol designed to expose the exercising limb to the desired level of BFR in a progressive fashion. Thus, before inflating the pneumatic cuff to the target pressure, an adaptive cycle of cuff inflation/deflation was performed, 


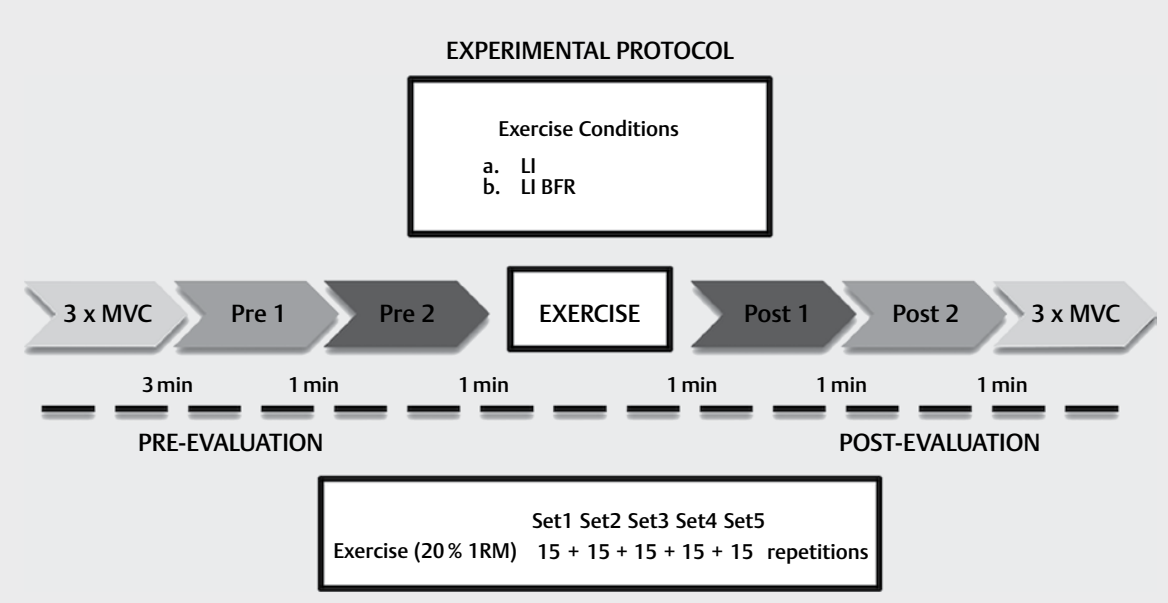

- Fig. 1 Experimental protocol. All participants performed 5 sets of unilateral knee extensions at $20 \%$ of one repetition maximum (1RM) $(15+15+15+15+15$ reps). 30 s of passive rest were allowed between sets. Blood flow restriction (BFR) was set at $60 \%$ of the arterial occlusion pressure for the right lower limb. Three maximal voluntary contractions (MVCs) were completed before and after exercise ( 1 min of rest between each MVC). MVC was defined as the highest force value obtained during testing. Isometric target-force contractions (trapezoidal force profile) were performed once at $40 \%$ MVC during pre 1, pre 2, post 1 and post 2. Surface electromyographic signal, recorded during each submaximal contraction, was subsequently decomposed to extract the motor unit action potential trains of concurrently active motor units.

with inflations of 30 s and deflations of 10 s executed gradually at 50 , 75 , and $100 \%$ of each target point. The cuff was inflated to the target pressure immediately at the beginning of pre 2 . Then, it was deflated immediately at the beginning of post 2 . BFR was sustained for a total of $\sim 8$ min per session. For safety reasons, a pulse oximeter (Onyx ${ }^{\circledR}$ || 9560, Nonin Medical Inc., Plymouth, MN, USA) was used (placed on the right hallux) to ensure that blood flow was not completely halted by tissue edema immediately after each set of BFR exercise.

\section{Target-force trajectory at $40 \%$ of maximal voluntary contraction}

Measurements were taken during submaximal voluntary contractions performed at each time point (pre 1, pre 2, post 1 and post 2). Participants were instructed to produce an isometric trapezoidal force profile (presented on a computer screen) with a plateau at $40 \%$ MVC. The force profile had the following characteristics: (1) initial quiescent region with $3 \mathrm{~s}$ of duration, (2) up-ramp contraction with a slope of $6.5 \% \mathrm{MVC} / \mathrm{s},(3)$ constant target-force region at $40 \% \mathrm{MVC}$ with $12 \mathrm{~s}$ of duration and (4) down-ramp contraction with a slope of $6.5 \% \mathrm{MVC} / \mathrm{s}$.

\section{EMG and force signal acquisition}

EMG signals were synchronized with the force signal recorded from a load cell (SM S-Type; Interface, Scottsdale, AZ, USA), specifically adapted for the leg extension machine. Surface EMG was recorded from the vastus lateralis muscle using a sensor array (DelSys Inc., Boston, MA, USA) consisting of five cylindrical pins ( $0.5 \mathrm{~mm}$ diameter) located on the corners and the center of a $5 \times 5 \mathrm{~mm}$ square. Pairwise differentiation of the 5 electrodes yielded $4 \mathrm{EMG}$ channels that were band-pass filtered between 20 and $450 \mathrm{~Hz}$. Both the EMG sensor and reference electrode were connected to a Delsys Bagnoli-16 amplifier (total gain of 1000) and to an analog-to-digital converter with a sampling frequency of $20 \mathrm{kHz}$ (USB-6251; National Instruments, Austin, TX, USA). EMGworks and dEMG software (Delsys Inc.) were used for data acquisition and EMG signal decomposition, respectively. The four channels of raw EMG were analyzed simultaneously, using dEMG algorithms, to extract the MUAP trains of concurrently active MUs [9]. The accuracy of each decomposed MUAP train was then calculated [23]. We analyzed only MUAP trains that were decomposed with an accuracy level >90\% [12]. The firing behavior of each MU was characterized by exploring the following parameters: (1) MU recruitment threshold, calculated as the force level at which the MU started firing; (2) MU mean firing rate, calculated from the inverse of the inter-pulse intervals in a 4-s period between 18-22 s of the constantforce segment of each submaximal contraction, and (3) the MUAP shape amplitude, calculated as the maximum amplitude of the positive or negative MUAP phases detected across the four EMG channels [12].

\section{Statistical analysis}

Data were reported as mean \pm SD. Mauchly's test was used to test the assumption of sphericity. The Greenhouse-Geisser correction was implemented to adjust the degrees of freedom for the averaged tests of significance when the assumption of sphericity was not met. Paired t tests were used to explore differences in MVC between conditions (LI vs. LI BFR) before and after exercise. Regression lines of the relationship between MU recruitment threshold and firing rate (RT/FR) were calculated from the EMG signal. A twoway repeated measures ANOVA [ 2 conditions (LI vs. LI BFR) x 4 times (pre 1 vs. pre 2 vs. post 1 vs. post 2)] was computed to determine the effects of each condition on the relationship between MU recruitment threshold and firing rate (linear slope coefficients and yintercepts). Post hoc t tests, with Bonferroni's adjustment, were used for all repeated measures analyses when significant effects were detected. The relationship between MU shape amplitude and 
firing rate (SA/FR) was non-linear. Thus, inverse exponential regressions of the form $\mathrm{y}=\mathrm{A}+\mathrm{Be} \mathrm{e}^{(-\mathrm{Cx})}$ were used in this case. All statistical calculations were computed using the SPSS (version 22.0, SPSS Inc., Chicago, IL, USA) and significance was set at $\mathrm{p}<0.05$.

\section{Results}

MVC decreased by $20.5 \pm 10.1 \%$ after exercise, but only in the $\mathrm{LI}$ BFR condition $(t=5.3, p=0.001)$. In the $L I$ condition, MVC had a non-significant decrease of $0.2 \pm 4.1 \%$.

\section{Recruitment threshold/firing rate analysis}

There was a condition-by-time interaction for the RT/FR linear slope coefficient $(F=10.7, p=0.002)$. Post hoc analyses indicated no differences between conditions in transition from pre 1 to pre 2, nor from post 1 to post 2 . In contrast, as can be seen in $>$ Table 1 , the magnitude of decrease in the RT/FR linear slope coefficient from pre 2 to post 1 was of smaller magnitude in LI when compared to that seen in LI BFR $[-44.4 \pm 33.1$ ( $p=0.004)$ vs. $-165.1 \pm 120.4 \%$ $(p=0.001)$, respectively]. $>$ Fig. 2 a shows the linear regressions between conditions in 2 representative participants. As depicted in $\mathbf{F i g}$. 2b, similar results were also obtained for comparisons between pre 1 and post 2 [LI: $-72.8 \pm 56.8$ ( $p=0.013)$ vs. LI BFR: $-234.0 \pm 133.0 \%(p=0.001)]$.
There was a condition-by-time interaction for the $y$-intercept $(F=7.2, p=0.012)$. Follow-up t tests revealed no differences between conditions neither from pre 1 to pre 2 nor from pre 2 to post 1. Conversely, there was an increase in the $y$-intercept values from post 1 to post 2, but this occurred only after LI BFR exercise $(+24.1 \pm 17.6 \%, p=0.005)$.

\section{Shape amplitude/firing rate analysis}

Qualitatively, the SA/FR regression line was considerably altered by the LI BFR condition. Specifically, there was an upward-right shift between pre 2 and post 1, thus indicating that MUs with similar MUAP amplitude increased their firing rates and that LI BFR elicited the recruitment of MUs with higher MUAP amplitudes. - Fig. 3 shows the comparison between conditions in 4 representative participants.

\section{Discussion}

It is often argued that BFR enhances the recruitment of high threshold MUs during $\mathrm{LI}$ exercise and that, despite low mechanical loading, this triggers muscle hypertrophy [4, 6, 8, 24-26]. However, to our knowledge, the impact of acute LI BFR exercise on the firing behaviour of individual MUs has never been determined. Using surface EMG signal decomposition, we found that LI BFR exercise induces a greater decrease in the linear slope coefficient of the relation between MU recruitment threshold and firing rate. This

- Table 1 Individual linear slope coefficient and y-intercept values for pre 2 and post 1. Pre- to post-exercise mean values \pm standard deviation (SD) for lowintensity (LI) exercise with and without blood flow restriction (BFR).

\begin{tabular}{|c|c|c|c|c|c|c|c|c|}
\hline \multirow{5}{*}{$\frac{\text { Participant }}{1}$} & \multicolumn{8}{|c|}{ Pre 2 to Post 1} \\
\hline & \multicolumn{4}{|c|}{ Linear Slope Coefficient } & \multicolumn{4}{|c|}{ y-Intercept } \\
\hline & \multicolumn{2}{|c|}{ LI } & \multicolumn{2}{|c|}{ LI BFR } & \multicolumn{2}{|c|}{ LI } & \multicolumn{2}{|c|}{ LI BFR } \\
\hline & -0.29 & -0.32 & -0.27 & -0.66 & 19.5 & 17.3 & 21.1 & 24.4 \\
\hline & \multicolumn{2}{|c|}{+9.1} & \multicolumn{2}{|c|}{+146.1} & \multicolumn{2}{|c|}{-11.0} & \multicolumn{2}{|c|}{+15.6} \\
\hline \multirow[t]{2}{*}{2} & -0.5 & -0.53 & -0.61 & -1.67 & 23.8 & 24.1 & 25.8 & 33.7 \\
\hline & \multicolumn{2}{|c|}{+6.8} & \multicolumn{2}{|c|}{+173.9} & \multicolumn{2}{|c|}{+1.3} & \multicolumn{2}{|c|}{+30.4} \\
\hline \multirow[t]{2}{*}{3} & -0.31 & -0.56 & -0.18 & -0.98 & 21.8 & 22.3 & 17.5 & 21.4 \\
\hline & \multicolumn{2}{|c|}{+83.2} & \multicolumn{2}{|c|}{+447.7} & \multicolumn{2}{|c|}{+2.3} & \multicolumn{2}{|c|}{+22.7} \\
\hline \multirow[t]{2}{*}{4} & -0.22 & -0.38 & -0.4 & -1.26 & 17.8 & 18.8 & 21.6 & 20.5 \\
\hline & \multicolumn{2}{|c|}{+73.1} & \multicolumn{2}{|c|}{+216.9} & \multicolumn{2}{|c|}{+5.7} & \multicolumn{2}{|c|}{-5.1} \\
\hline \multirow[t]{2}{*}{5} & -0.37 & -0.72 & -0.76 & -1.13 & 19.8 & 23.0 & 24.3 & 26.5 \\
\hline & \multicolumn{2}{|c|}{+94.5} & \multicolumn{2}{|c|}{+49.3} & \multicolumn{2}{|c|}{+16.2} & \multicolumn{2}{|c|}{+8.8} \\
\hline \multirow[t]{2}{*}{6} & -0.52 & -0.59 & -0.62 & -0.85 & 21.7 & 25.5 & 25.6 & 28.7 \\
\hline & & & & & & & & \\
\hline 7 & -1.01 & -1.27 & -0.89 & -1.95 & 33.8 & 34.6 & 33.8 & 32.7 \\
\hline & & & & & & & & \\
\hline 8 & -0.49 & -0.72 & -0.43 & -0.99 & 23.6 & 23.7 & 21.9 & 21.5 \\
\hline & & & & & & & & \\
\hline Mean & -0.46 & -0.64 & -0.52 & -1.19 & 22.7 & 23.7 & 23.8 & 26.2 \\
\hline SD & 0.23 & 0.27 & 0.23 & 0.4 & 4.6 & 4.9 & 4.2 & 4.8 \\
\hline Diff\% & & & & & & & & \\
\hline & & & & & & & & \\
\hline $\begin{array}{l}\text { Linear slope } \\
\text { firing rate. P } \\
{ }^{*} \text { Differences }\end{array}$ & $\begin{array}{l}\text { cients a } \\
\text { dd post } \\
\text { n condi }\end{array}$ & $\begin{array}{l}\text { values } \\
\text { nts wer } \\
\text { ** Dif }\end{array}$ & in each & $\begin{array}{l}\text { gressic } \\
\text { nd LI B }\end{array}$ & $\begin{array}{l}\text { etwee } \\
\text { befor }\end{array}$ & $\begin{array}{l}\text { (MU) } \\
\text { r afte }\end{array}$ & $\begin{array}{l}\text { hent } t \\
\text { e, res }\end{array}$ & id me \\
\hline
\end{tabular}


MU Recruitment Threshold by MU Firing Rate Pre2 to Post1

LI
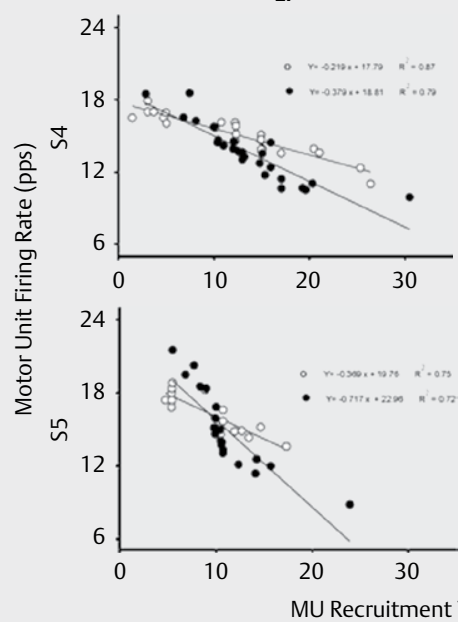

- Pre2 • Post1
LI BFR
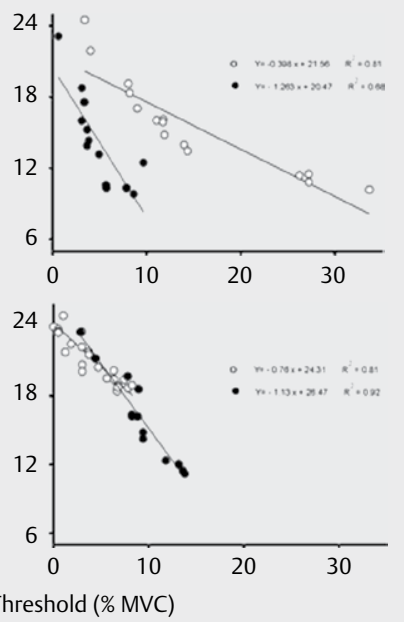

Linear Slope Coefficient MU Recruitment Threshold by Firing Rate Pre1 to Post2

- Fig. 2 Relationship between motor unit (MU) recruitment threshold and mean firing rates obtained during a pre 2 and post 1 , and during $\mathbf{b}$ pre 1 and post 2. a Relationship between MU recruitment threshold and mean firing rates in two representative participants. All MUs were plotted for low-intensity (LI) exercise performed with and without blood flow restriction (BFR). b Changes in individual and mean linear slope coefficients were plotted between conditions in all participants (LI exercise with and without BFR). Pre 1 and post 2 were measured without BFR. MVC $=$ maximal voluntary contraction. ${ }^{*}$ Differences within conditions, $\mathrm{p}<0.05 .{ }^{*}{ }^{*}$ Differences between conditions, $\mathrm{p}<0.05$.

suggests that acute LI BFR exercise is effective for heightening the recruitment of MUs with higher threshold and lower firing rates $[12,23,27]$. In addition, the magnitude of increase in the RT/FR $y$-intercept from post 1 to post 2 (i. e., following cuff release) was more expressive in the LI BFR condition. This further indicates that MUs recruited at similar force levels fire at higher rates during muscle contractions performed post-LI BFR exercise. Finally, we showed that LI BFR exercise elicits an upward right shift of the SA/FR relationship. This provides preliminary evidence that performing this type of resistance exercise is effective for recruiting MUs with higher amplitude. It also suggests that MUs with similar MUAP amplitude fire at higher rates after LI BFR exercise.

\section{Mechanical output}

MVC decreased only after LI BFR exercise. These results are consistent with past research $[1,3,28,29]$ and most likely reflect neuromuscular fatigue instead of muscle damage [19]. Nevertheless, one previous report has shown that, when performed at $60 \% \mathrm{BFR}, \mathrm{LI}$ exercise does not exacerbate neuromuscular fatigue [18]. However, in that study, participants exercised on an isokinetic dynamometer and muscle loading was limited to the concentric phase of the lift. Conversely, in the present study, muscle loading was extensive to both phases of motion (i. e., concentric and eccentric) and this likely explains the discrepancies between studies.

\section{Effects of BFR (pre 1 to pre 2)}

There were no changes in the linear slope coefficients or the $y$-intercepts from pre 1 to pre 2 in either condition. Thus, our findings show that neuromuscular function is not affected by relative BFR per se and this corroborates past findings. Specifically, there is compelling evidence that the attenuation of both muscle atrophy and declines in strength with brief applications of BFR are mediated via an acute fluid shift-induced increase in muscle size [30].

\section{Effects of LI BFR exercise (pre 2 to post 1 )}

The analysis of the RT/FR regression lines indicates that the linear slope coefficient was the only parameter altered by LI exercise (either with or without BFR). Importantly, both conditions induced significant decrements in its values and this might be secondary to the additional recruitment of high-threshold MUs during the ramp contractions post-LI exercise $[11,12]$. According to the "onion skin" principle, these higher-threshold MUs have lower firing rates, thus determining a more negative linear slope coefficient between RT/ FR $[12,23,27]$. Nevertheless, we found that the impact of LI BFR exercise on decreasing the linear slope coefficient was four times larger than that seen post-LI. This is similar to that reported in past research for other fatiguing-tasks and strongly suggests that LI BFR is particularly effective in decreasing the recruitment threshold of all motor units $[11,12]$. The progressive recruitment of new MU may also provide a partial explanation for this effect [11, 12, 14-16]. There are at least two hypothetical reasons for such findings: (1) the metabolic stress resulting from exercise performed with reduced blood flow and peripheral venous pooling, which might trigger the recruitment of additional glycolytic MUs while preventing the removal of metabolites from the muscles [21,31]; (2) the engagement of extra MUs, which might represent a compensatory 


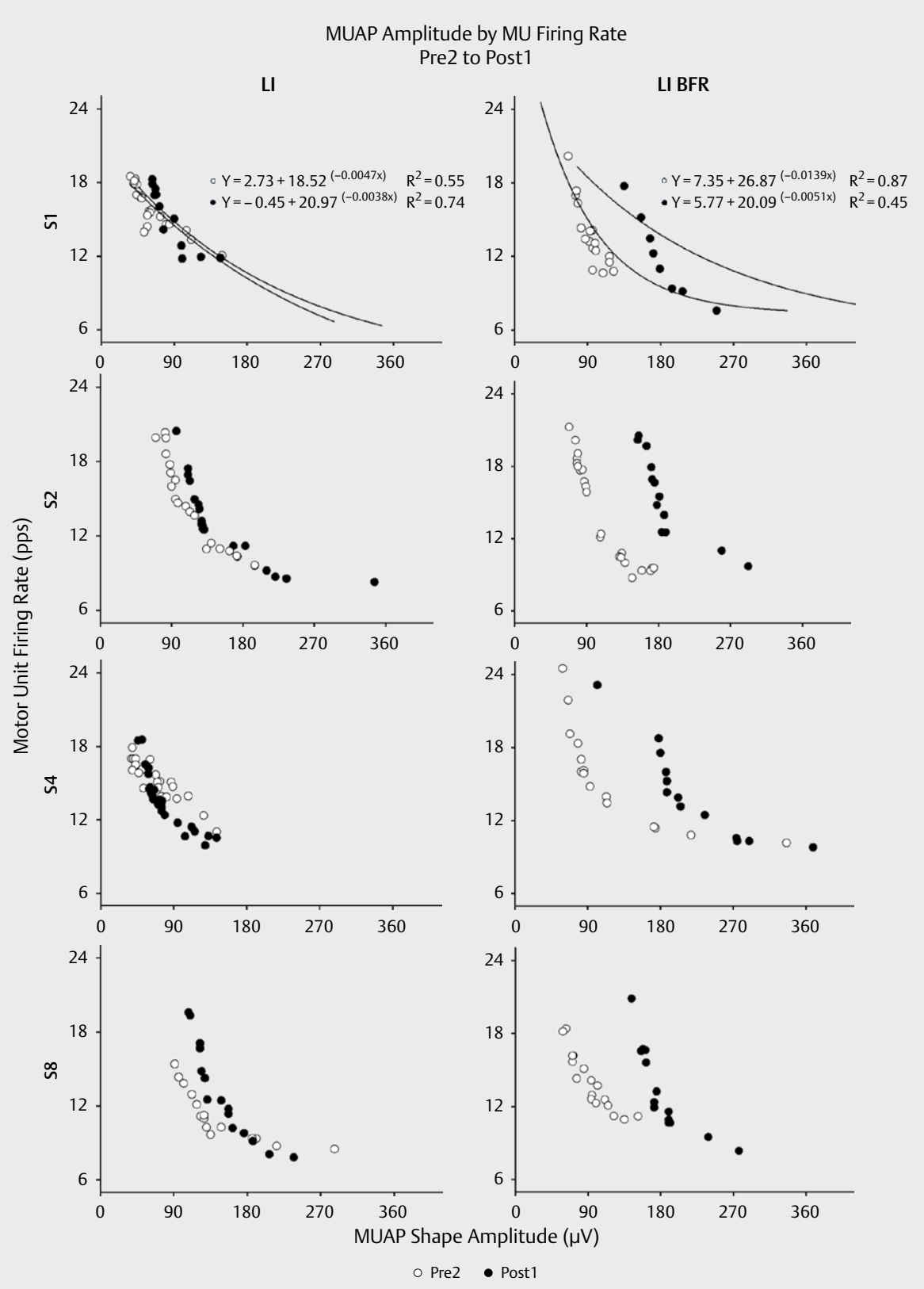

- Fig. 3 Representative participant-specific relations between motor unit (MU) mean firing rates and action potential (MUAP) amplitude, obtained during pre 2 and post 1 . All motor units were plotted for low-intensity (LI) exercise a without and $\mathbf{b}$ with blood flow restriction (BFR).

strategy to preserve mechanical output after fatiguing LI BFR exercise $[11,13-15]$. Our data on the SA/FR regression lines further indicate that the MU recruited in the LI BFR condition exhibited higher MUAP amplitudes. Taken together, our findings support the contention that acute LI BFR exercise promotes the activation of high-threshold MUs [32].

\section{Effects of BFR release (post 1 to post 2)}

Cuff release post-Ll exercise heightened the magnitude of increase in the $y$-intercept of the RT/FR regression lines. This is compatible with an increased MU firing rate post-BFR release, possibly to maintain force production within the context of BFR-induced fatigue, which was quantified through the decrease in mechanical output after LI BFR exercise. Importantly, because there were no differences between conditions for the RT/FR linear slope coefficient, our data suggest that MU recruitment was similar between conditions in transition from post 1 to post 2. Compared to that seen post-exercise before BFR release, this represents a different neurophysiological strategy of responding to the same testing paradigm (submaximal isometric tracking task). 


\section{Conclusion}

This is the first study to compare the individual changes in MU activation (through EMG decomposition) between LI exercise performed with and without BFR. We found that BFR induces a shift in the $\mathrm{MU}$ recruitment pattern during $\mathrm{LI}$ exercise, and this is novel. Specifically, there is an early recruitment of MU with high threshold and low firing rates. Moreover, we demonstrated that the magnitude of this effect is much larger post-LI BFR compared to that seen post-Ll exercise. Finally, we showed that the firing rate of similarly sized MUs increases after BFR release. Further research is warranted to determine whether these effects underlie the hypertrophy gains with BFR.

\section{Limitations}

One important limitation of surface EMG decomposition is that the population of tracked MUs can differ between experimental sessions, despite consistent EMG electrode placement. This may also apply to different measurements taken within the same testing session (due to differences in skin blood flow, blood pooling or even tissue filtering that might occur both with and without BFR). While acknowledging that this might represent a limitation of our methodological approach, it is important to note that we were able to decompose a relatively high number of MUs in each measurement time-point contraction (i. e., 21 MUs for LI and 17 for LI BFR). For this reason, we believe that our interpretations on the interaction between fatigue and altered MU firing behavior are correct [12, 23]. Moreover, within the context of BFR, the use of surface EMG decomposition raises the possibility that MUAP amplitudes might have been diminished by the impact of edema on signal quality. Nevertheless, the algorithms used for signal decomposition have been extensively tested in past research for: (1) changes of MUAP shape under a variety of conditions, (2) superpositions of action potentials, (3) a large dynamic range in the amplitudes among the MUAPs, and (4) similar MUAPs shapes at various times [33]. Thus, we are confident that our findings were not substantially affected by this limitation.

\section{Acknowledgements}

This work was supported by funds derived the Foundation for Science and Technology (FCT), Portugal: (1) Unit I\&D 472 (UID/ DTP/00447/2019) and (2) (PTDC/DTP-DES/5714/2014). The authors would like to express their gratitude to Dr. Paola Contessa (Delsys Inc., Natick, USA), for all the invaluable assistance, comments and remarks.

\section{Conflict of Interest}

Authors declare that they have no conflict of interest.

\section{References}

[1] Cook SB, Murphy BG, Labarbera KE. Neuromuscular function after a bout of low-load blood flow-restricted exercise. Med Sci Sports Exerc 2013; 45: 67-74
[2] Fahs CA, Loenneke JP, Thiebaud RS, Rossow LM, Kim D, Abe T, Beck TW, Feeback DL, Bemben DA, Bemben MG. Muscular adaptations to fatiguing exercise with and without blood flow restriction. Clin Physiol Funct Imaging 2015; 35: 167-176

[3] Karabulut M, Cramer JT, Abe T, Sato Y, Bemben MG. Neuromuscular fatigue following low-intensity dynamic exercise with externally applied vascular restriction. J Electromyogr Kinesiol 2010; 20: 440-447

[4] Moritani T, Sherman WM, Shibata M, Matsumoto T, Shinohara M. Oxygen availability and motor unit activity in humans. Eur J Appl Physiol Occup Physiol 1992; 64: 552-556

[5] Shinohara M, Kouzaki M, Yoshihisa T, Fukunaga T. Efficacy of tourniquet ischemia for strength training with low resistance. Eur J Appl Physiol Occup Physiol 1998; 77: 189-191

[6] Takarada Y, Nakamura Y, Aruga S, Onda T, Miyazaki S, Ishii N. Rapid increase in plasma growth hormone after low-intensity resistance exercise with vascular occlusion. J Appl Physiol (1985) 2000; 88: 61-65

[7] Takarada Y, Sato Y, Ishii N. Effects of resistance exercise combined with vascular occlusion on muscle function in athletes. Eur J Appl Physiol 2002; 86: 308-314

[8] Moore DR, Burgomaster KA, Schofield LM, Gibala MJ, Sale DG, Phillips SM. Neuromuscular adaptations in human muscle following low intensity resistance training with vascular occlusion. Eur J Appl Physiol 2004; 92: 399-406

[9] De Luca C], Adam A, Wotiz R, Gilmore LD, Nawab SH. Decomposition of surface EMG signals. J Neurophysiol 2006; 96: 1646-1657

[10] Nawab SH, Chang SS, De Luca C]. High-yield decomposition of surface EMG signals. Clin Neurophysiol 2010; 121: 1602-1615

[11] Adam A, De Luca C]. Recruitment order of motor units in human vastus lateralis muscle is maintained during fatiguing contractions. J Neurophysiol 2003; 90: 2919-2927

[12] Contessa P, De Luca C], Kline JC. The compensatory interaction between motor unit firing behavior and muscle force during fatigue. J Neurophysiol 2016; 116: 1579-1585

[13] Bigland-Ritchie BR, Dawson NJ, Johansson RS, Lippold OC. Reflex origin for the slowing of motoneurone firing rates in fatigue of human voluntary contractions. J Physiol 1986; 379: 451-459

[14] Carpentier A, Duchateau J, Hainaut K. Motor unit behaviour and contractile changes during fatigue in the human first dorsal interosseus. J Physiol 2001; 534: 903-912

[15] Enoka RM, Robinson GA, Kossev AR. Task and fatigue effects on low-threshold motor units in human hand muscle. J Neurophysiol 1989; 62: 1344-1359

[16] Garland S], Enoka RM, Serrano LP, Robinson GA. Behavior of motor units in human biceps brachii during a submaximal fatiguing contraction. J Appl Physiol (1985) 1994; 76: 2411-2419

[17] Harriss DJ, Macsween A, Atkinson G. Standards for ethics in sport and exercise science research: 2018 Update. Int J Sports Med 2017; 38: 1126-1131

[18] Fatela P, Reis JF, Mendonca GV, Avela J, Mil-Homens P. Acute effects of exercise under different levels of blood-flow restriction on muscle activation and fatigue. Eur J Appl Physiol 2016; 116: 985-995

[19] Loenneke JP, Abe T. Does blood flow restricted exercise result in prolonged torque decrements and muscle damage? Eur J Appl Physiol 2012; 112: 3445-3446 author reply 3447-3449

[20] Yasuda T, Brechue WF, Fujita T, Sato Y, Abe T. Muscle activation during low-intensity muscle contractions with varying levels of external limb compression. J Sports Sci Med 2008; 7: 467-474

[21] Yasuda T, Brechue WF, Fujita T, Shirakawa J, Sato Y, Abe T. Muscle activation during low-intensity muscle contractions with restricted blood flow. J Sports Sci 2009; 27: 479-489 
[22] Yasuda T, Ogasawara R, Sakamaki M, Ozaki H, Sato Y, Abe T. Combined effects of low-intensity blood flow restriction training and high-intensity resistance training on muscle strength and size. Eur J Appl Physiol 2011; 111: 2525-2533

[23] De Luca C], Contessa P. Hierarchical control of motor units in voluntary contractions. J Neurophysiol 2012; 107: 178-195

[24] Krustrup P, Soderlund K, Relu MU, Ferguson RA, Bangsbo J. Heterogeneous recruitment of quadriceps muscle portions and fibre types during moderate intensity knee-extensor exercise: Effect of thigh occlusion. Scand J Med Sci Sports 2009; 19: 576-584

[25] Meyers R. Does blood flow restriction enhance hypertrophic signaling in skeletal muscle? J Appl Physiol (1985) 2006; 100: 1443-1444

[26] Yasuda T, Abe T, Sato Y, Midorikawa T, Kearns C, Inoue K, Ryushi T, Muscle NI. fiber cross-sectional area is increased after two weeks of twice-daily KAATSU resistance training. Int J KAATSU Training Res 2005; 1: 65-70

[27] Holobar A, Farina D, Gazzoni M, Merletti R, Zazula D. Estimating motor unit discharge patterns from high-density surface electromyogram. Clin Neurophysiol 2009; 120: 551-562
[28] Cook SB, Clark BC, Ploutz-Snyder LL. Effects of exercise load and bloodflow restriction on skeletal muscle function. Med Sci Sports Exerc 2007; 39: 1708-1713

[29] Loenneke JP, Kim D, Fahs CA, Thiebaud RS, Abe T, Larson RD, Bemben DA, Bemben MG. Effects of exercise with and without different degrees of blood flow restriction on torque and muscle activation. Muscle Nerve 2015; 51: 713-721

[30] Loenneke JP, Fahs CA, Thiebaud RS, Rossow LM, Abe T, Ye X, Kim D, Bemben MG. The acute muscle swelling effects of blood flow restriction. Acta Physiol Hung 2012; 99: 400-410

[31] Loenneke JP, Fahs CA, Rossow LM, Abe T, Bemben MG. The anabolic benefits of venous blood flow restriction training may be induced by muscle cell swelling. Med Hypotheses 2012; 78: 151-154

[32] De Luca C], Erim Z. Common drive of motor units in regulation of muscle force. Trends Neurosci 1994; 17: 299-305

[33] Nawab SH, Wotiz RP, De Luca C]. Improved resolution of pulse superpositions in a knowledge-based system for EMG decomposition. In, Twenty-sixth International Conference of the IEEE Engineering in Medicine and Biology Society. San Francisco 2004; 69-71 